\title{
Chemikalien-Syndrome: Hilfe vom Psychologen?
}

Kopfschmerzen, Konzentrationsstörungen, Nervosität, Schlaflosigkeit und Abgeschlagenheit - dies sind die typischen Anzeichen für unspezifische Syndrome wie Multiple Chemical Sensitivity (MCS), Öko-Syndrom, Chronic Fatigue Syndrom (CFS) oder Sick Building Syndrom (SBS). In der Diskussion steht nach wie vor, ob die betroffenen Patienten wirklich überempfindlich reagieren und welche Rolle die Psyche dabei spielt.

Typisch für die Symptome der Chemikalien-Syndrome sind die in der Regel unspezifischen und meist auch nicht objektivierbaren Beschwerden. Auch liegen die Schadstoffkonzentrationen, bei denen die Symptomatik auftritt, häufig unterhalb der Grenzwerte - also in einem Bereich, den die Mehrheit der Bevölkerung problemlos toleriert.

Deshalb tun sich Toxikologen wie auch Allergologen mit einer wissenschaftlichen Erklärung für dieses Krankheitsbild schwer, ein kausaler Zusammenhang zwischen Dosis und Wirkung ist in diesem Fall nicht herzustellen. Ist also alles nur ein Produkt der Psyche?

Auffällig ist jedenfalls, daß vermehrt Patienten mit psychiatrischen Erkrankungen an Chemikalien-Syn-

\section{Objektive Diagnose noch nicht möglich}

Hinter dem Etikett „Chemikalien-Syndrome“ können sich verschiedene Erkrankungen verbergen: Allergien und Pseudo-Allergien, aber auch Hormonstörungen, Autoimmmunerkrankungen oder psychische bzw. psychosomatische Störungen. Eine interdisziplinäre Diagnosestellung ist deshalb bei dieser unspezifischen Symptomatik unbedingt erforderlich. dromen leiden, wie u.a. Th. Zilker, München, in seinen Untersuchungen feststellte. Umweltmediziner erklären MCS dagegen mit einer angeborenen oder erworbenen erhöhten Empfindlichkeit gegenüber Chemikalien (Definition siehe nebenstehender Kasten).

$\mathrm{Ob}$ die Symptome nun biogen oder psychogen sind - der Arzt sollte den Patienten und seine Symptomatik auf jeden Fall ernst nehmen. Nur so läßt sich Doctor-Hopping und vor allem das Abwandern der Patienten in die Alternativmedizin, was gerade unter den MCS-Patienten weit verbreitet ist, verhindern.

\section{MCS - erlerntes Verhalten durch Konditionierung über die Nase}

Einen neuen und interessanten Therapieansatz stellte U. Strehl, Institut für Medizinische Psychologie und Verhaltenstherapie der Universität Tübingen, vor. Sie betrachtet MCS als ein Verhaltensmuster, das irgendwann erlernt wurde und deshalb auch wieder verlernt werden kann.

Eine besondere Rolle beim Erlernen eines solchen Verhaltens durch klassische Konditionierung spielt anscheinend der Geruchssinn. Ein Indiz hierfür liefert die Tatsache, daß MCSauslösende Stoffe häufig sehr geruchsintensiv sind. Bleiverbindungen - also in der Regel geruchlose Substanzen verursachen dagegen kein MCS.

Um die entsprechenden Verhaltensmuster wieder zu löschen, bieten sich eine Reihe verhaltenstherapeutischer
Kriterien der Multiple Chemical Sensitivity

Von einer Multiple Chemical Sensitivity (MCS) spricht man (nach Cullen), wenn der Patient über Symptome klagt, die

durch nachweisbare Schadstoffexposition ausgelöst werden,

- mehr als ein Organsystem betreffen,

- bei vorhersehbaren Reizen auftreten,

- durch verschiedenste Chemikalien schon bei niedriger Konzentration ausgelöst werden,

- sich nicht durch die üblichen Organfunktionstests erfassen lassen.

Methoden an. Dazu zählen Desensibilisierung, Reizkonfrontation in Kombination mit Entspannungsverfahren, Biofeedback, Aufbau alternativen Verhaltens sowie kognitive Umstrukturierung.

Hat ein Patient beispielsweise das Gefühl: „Das halte ich nicht aus“, so soll er mit Hilfe dieser Methoden lernen zu denken und zu sagen: „Ich kann es ja mal probieren". Ein weiteres Lernziel ist, pathophysiologische Reaktionen wie z.B. Herzrasen, Blutdruckanstiege oder Schwindelgefühle selbst zu regulieren.

\section{Verhaltenstherapie als Ausweg -} bisher aber nur selten angewendet Verhaltenstherapeutische Maßnahmen wurden zwar bei Chemikalien-Syndromen noch selten angewendet, doch belegt ein Beispiel aus der Literatur ihre Wirksamkeit. Guglielmi et al. verhalfen einer 52jährigen MCS-Patienten damit wieder zu einer nahezu normalen Lebensführung, obwohl sie vorher nur noch mit Schutzmaske Zeitung lesen konnte, weil sie sogar gegen die Druckerschwärze sensibel reagierte (Journal of Behavioral Therapy and Experimental Psychiatry 1994; 25: 197-209).

(jn)

Tagung „Chemikalien-Syndrome - Fiktion oder Wirklichkeit“, veranstaltet vom Institut für Toxikologie und Umweltmedizin der Technischen Universität München, 1999. 